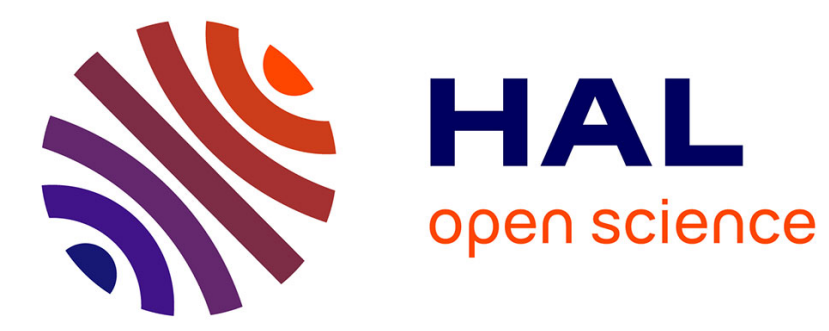

\title{
Effect of sodium concentration on the synthesis of faujasite by two-step synthesis procedure
}

Lei Zhao, Ge Yang, Hailing Guo, Chunzheng Wang, Lijuan Wang, Svetlana Mintova

\section{- To cite this version:}

Lei Zhao, Ge Yang, Hailing Guo, Chunzheng Wang, Lijuan Wang, et al.. Effect of sodium concentration on the synthesis of faujasite by two-step synthesis procedure. Chemical Research in Chinese Universities, 2021, 37 (5), pp.1137-1142. 10.1007/s40242-021-1292-2 . hal-03414956

\section{HAL Id: hal-03414956 https://hal.science/hal-03414956}

Submitted on 4 Nov 2021

HAL is a multi-disciplinary open access archive for the deposit and dissemination of scientific research documents, whether they are published or not. The documents may come from teaching and research institutions in France or abroad, or from public or private research centers.
L'archive ouverte pluridisciplinaire HAL, est destinée au dépôt et à la diffusion de documents scientifiques de niveau recherche, publiés ou non, émanant des établissements d'enseignement et de recherche français ou étrangers, des laboratoires publics ou privés. 


\title{
Effect of sodium concentration on the synthesis of faujasite by two-step synthesis procedure
}

Lei Zhao ${ }^{\mathrm{a}}$, Ge Yang ${ }^{\mathrm{a}}$, Hailing Guo ${ }^{\mathrm{a}, *}$, Chunzheng Wang ${ }^{\mathrm{a}}$, Lijuan Wang ${ }^{\mathrm{a}}$ and Svetlana Mintova ${ }^{\mathrm{a}, \mathrm{b}}, *$

a: State Key Laboratory of Heavy Oil Processing, Key Laboratory of Catalysis, China National Petroleum Corp. (CNPC) China University of Petroleum (East China), Qingdao 266555, P. R. China E-mail: guohl@upc.edu.cn; mintova@ensicaen.fr

b: Laboratoire Catalyse et Spectrochimie (LCS), CNRS, ENSICAEN, Université de Caen, 6 boulevard du Maréchal Juin, 14050 Caen France.

\begin{abstract}
The relationship between concentration of sodium cations and the properties of faujasite (FAU) zeolite using a two-step synthesis procedure including (1) formation of amorphous aluminosilicate precursors and separation of amorphous nanoparticles, and (2) transformation of these amorphous particles into zeolite crystals by treatment with alkali solutions $(\mathrm{NaOH})$ were studied. Three representative samples including two nano-sized zeolites and one micron-sized zeolite were prepared using different concentration of sodium hydroxide. The crystallization process of these zeolites was studied in details by FTIR, NMR, XRD, SEM, TEM, $\mathrm{N}_{2}$ adsorption. The results indicated that minor changes in the concertation of inorganic cations can significantly shorten the induction period and crystallization time and thus affect the morphology, size and chemical composition of the zeolite crystals.
\end{abstract}

Keywords: synthesis; zeolite; morphology; alkali metal

\section{Introduction}

The synthesis of zeolites continues to receive attentions due to its great industrial value in catalysis, adsorption, separation, medicine, food, etc ${ }^{[1-5]}$. Organic amine template, surfactant, seeding aid, inorganic cation ${ }^{[6-9]}$ are widely used in the preparation of zeolites. Among them, the inorganic structural direction agents (single or mixtures of alkali cations) are highly desired due to their green nature and low cost. 
Therefore, efforts have been made to develop organic template-free methods to synthesize zeolites with emphasis on their particle size and chemical composition. Several inorganic ions (e.g. $\mathrm{Na}^{+}, \mathrm{K}^{+}, \mathrm{Li}^{+}, \mathrm{Cs}^{+}$) are introduced to prepared nanosized zeolite ${ }^{[10-15]} \cdot \mathrm{Na}^{+}$is commonly used in the synthesis of nanosized zeolites with EMT, LTA, FAU, SOD, MFI, MTW framework structures ${ }^{[16-20]}$. However, the effect of the inorganic ions on the nucleation and crystallization process of nanozeolites is still under debate. At present, the inorganic cations with higher charge density, which can compensate more $\mathrm{AlO}_{4}{ }^{-}$are used to synthesize zeolite with low $\mathrm{Si} / \mathrm{Al}$ ratio. Therefore, the concentration of sodium cations in the initial precursor mixtures is of importance and is considered to predetermine partially the $\mathrm{Si} / \mathrm{Al}$ ratio of final zeolites. However, the $\mathrm{Si} / \mathrm{Al}$ ratio of the zeolite is controlled by the whole reaction process ${ }^{[21]}$. The classical one-step hydrothermal synthesis promotes the simultaneous nucleation and crystallization in highly diluted colloidal precursor suspensions. But defining the role of each component in the reaction system and the entire sequence of events during the whole reaction process requires further study ${ }^{[22-23]}$. Recently we have reported on the two-step synthetic strategy for preparation of aluminosilicate amorphous precursor particles and their transformation into nanosized zeolites crystals ${ }^{[24]}$. In this paper, we focus on the synthesis of various zeolites with FAU type framework structure (zeolites $\mathrm{X}$ and $\mathrm{Y}$ ) by controlling precisely the formation and transformation of preformed amorphous nanosized particles into zeolites under treatment with alkali solutions. The influence of tiny variations of content of $\mathrm{NaOH}$ on the properties of zeolites such as morphology, size, $\mathrm{Si} / \mathrm{Al}$ ratio was studied.

\section{Materials and methods}

\subsection{Chemicals and reagents}

Aluminum powder (Al, 325 mesh, 99.5\%, Alfa Aesar), sodium hydroxide $(\mathrm{NaOH}$, Sinopharm, 97\%), colloidal silica $\left(\mathrm{SiO}_{2}\right.$, Ludox-HS30, 30 wt.\% $\mathrm{SiO}_{2}, \mathrm{pH}=9.8$, Aldrich), 1,3,5-triisopropylbenzene (Aladdin, 98\%), $\mathrm{NH}_{4} \mathrm{Cl}$ (Aladdin, $\mathrm{AR}$ ) were used as received.

\subsection{Preparation of amorphous precursor}

The amorphous precursor was prepared from a clear precursor suspension with a molar composition: $9 \mathrm{Na}_{2} \mathrm{O}: 0.59-0.7 \mathrm{Al}_{2} \mathrm{O}_{3}: 10 \mathrm{SiO}_{2}: 160 \mathrm{H}_{2} \mathrm{O}$. The initial reactants were mixed to prepare two initial solutions denoted A and B. Solution A was prepared 
by dissolving $10 \mathrm{~g}$ of $\mathrm{NaOH}$ in $20 \mathrm{~g}$ double distilled water $\left(\mathrm{dd}_{2} \mathrm{O}\right)$ followed by slow addition of 0.8-0.945 g aluminum powder. Solution B was prepared by mixing of $50 \mathrm{~g}$ colloidal silica with $8 \mathrm{~g} \mathrm{NaOH}$ and $17 \mathrm{~g}$ dd $\mathrm{H}_{2} \mathrm{O}$; as a result a turbid suspension was obtained. In order to obtain clear suspension, the container B was placed in an oven at $100{ }^{\circ} \mathrm{C}$ for 6 minutes. Solution A was added drop wise under vigorously stirring to the solution B; during the mixing, solution B was kept in ice. The resulting clear suspension was kept $24 \mathrm{~h}$ at room temperature for aging. Then the suspension was put into the freezer to remove the water with 46-48 g. Finally, the aluminosilicate precursor as white amorphous power was collected by centrifugation and washed several times with distilled water.

\subsection{Synthesis of FAU zeolite}

The $500 \mathrm{mg}$ amorphous power was mixed with $10 \mathrm{ml} \mathrm{NaOH}$ solution, the concentration of the $\mathrm{NaOH}$ solution varied from 0.5 wt.\% to 10 wt.\%. The suspensions were placed in an oven at $50{ }^{\circ} \mathrm{C}$ for $15 \mathrm{~h}, 36 \mathrm{~h}$ and $11 \mathrm{~d}$ different time. The crystalline materials were collected by centrifugation and washed several times with distilled water till $\mathrm{pH}$ of 7 . Samples are presented in Table 1.

\subsection{Ammonium exchange}

The samples FAU-1-nano, FAU-2-nano and FAU-3-micron were ion-exchanged with solution of $0.1 \mathrm{M} \mathrm{NH}_{4} \mathrm{Cl}$ at room temperature for 6 hours, washed with distilled water and calcined at $200{ }^{\circ} \mathrm{C}$.

\subsection{Characterizations}

Powder samples were measured using a Bruker D8 Advance diffractometer with $\mathrm{CuK} \alpha$ monochromatized radiation $(\lambda=1.5418 \AA)$. The crystal size, morphology and crystallinity of solids were determined by a scanning electron microscope (SEM) using a JEOL JSM-7900F and transmission electron microscope (TEM) using a FEI LaB6 TECNAI G230UT operated at $200 \mathrm{kV}$. FTIR spectra were recorded on a Bruker Vertex 70 spectrometer with an average of 64 scans. Nitrogen adsorption/desorption isotherms were measured using Quantachrome, Autosorb IQ, samples activated at 300 ${ }^{\circ} \mathrm{C}$ for $10 \mathrm{~h}$ with a heating rate $2{ }^{\circ} \mathrm{C} \cdot \mathrm{min}^{-1}$. The chemical composition was analyzed by inductively coupled plasma atomic emission spectrometer (ICPAES, a Leeman Labs Prodigy High Dispersion ICP instrument). TG-MS was performed using a 
Netzsch TG-MS (STA449 F5) instrument in a nitrogen atmosphere in the range of 50-1000 ${ }^{\circ} \mathrm{C}$ with a ramp of $2{ }^{\circ} \mathrm{C} \cdot \mathrm{min}^{-1} .{ }^{29} \mathrm{Si} \mathrm{NMR}$ spectra were recorded on a Bruker Avance $400(9.4 \mathrm{~T})$ spectrometer using $4 \mathrm{~mm}-\mathrm{OD}$ zirconia rotors and a spinning frequency of $12 \mathrm{kHz}$.

\subsection{Catalytic test}

The catalytic conversion of 1,3,5-triisopropylbenzene (TiPBz) was performed in a fixed bed reactor operating in a gas phase. For each experiment, catalyst $(50 \mathrm{mg})$ was loaded in the reactor and enclosed between two layers of quart sand (20-40 mesh). The catalyst was activated at $450{ }^{\circ} \mathrm{C}$ in $\mathrm{N}_{2}$ flow of $50 \mathrm{~mL} / \mathrm{min}$ for $1 \mathrm{~h}$ before reaction, and then the temperature was set to the to a reaction temperature of $350{ }^{\circ} \mathrm{C}$. The TiPBz was delivered using a carrier gas $\left(50 \mathrm{~mL} / \mathrm{min} \mathrm{N}_{2}\right)$ through a saturator containing $\mathrm{TiPBz}$ at $90{ }^{\circ} \mathrm{C}$. The reaction products were analyzed using an online gas chromatograph (Scion 456-GC GC 7890N) equipped with a flame ionization detector (FID).

\section{Results and discussion}

The amorphous nature of the solid precursor was confirmed by X-ray diffraction (Fig. 1a). As shown no diffraction peaks were found in the amorphous precursor noted as sample $\mathrm{S} 1$ in Table 1. This amorphous precursor was treated by 7 wt.\% $\mathrm{NaOH}$ solution at $50{ }^{\circ} \mathrm{C}$ for $15 \mathrm{~h}$ (sample S2, Table 1). The XRD pattern of this sample contains diffraction peaks belonging to FAU type framework structure. Further, the sample was characterized by ${ }^{29} \mathrm{Si}$ NMR and ICP. The results indicate that the sample is fully crystalline with $\mathrm{Si} / \mathrm{Al}=1.1$ that correspond to zeolite $\mathrm{X}$ (sample FAU-1-nano). While the addition of 2 wt.\% of $\mathrm{NaOH}$ to the amorphous solid precursor and crystallization at $50{ }^{\circ} \mathrm{C}$ for extended time $(36 \mathrm{~h}$,) resulted in a sample with $\mathrm{Si} / \mathrm{Al}$ of 1.5 (sample FAU-2-nano). Further, decreasing of the concentration of sodium hydroxide to $1 \mathrm{wt} . \%$ leads to slower crystal growth, and after HT treatment at $50{ }^{\circ} \mathrm{C}$ for $11 \mathrm{~d}$, a fully crystalline sample with a $\mathrm{Si} / \mathrm{Al}$ of 1.7 was obtained (sample FAU-3-micro, Table 1). It was found that the concentration of sodium cations is the growth-limiting nutrient in the formation of zeolite FAU. High concentration of sodium favors the crystallization rate and lead to the formation of zeolite with lower 
$\mathrm{Si} / \mathrm{Al}$ ratio. Sample FAU-3-micron required longer crystallization time in comparison to samples FAU-1-nano and FAU-2-nano. The intensity of diffraction peaks of sample FAU-1-nano is significantly low and they are broader indicating the smaller crystallite sizes. The average size of the crystals of sample FAU-1-nano is c.a. $25 \mathrm{~nm}$; this size was calculated using the Scherrer's equation based on the diffraction peaks $(2 \theta)$ at $6.11^{\circ}$ [111], $15.37^{\circ}$ [331], and $26.61^{\circ}$ [642]. The XRD pattern of sample FAU-3-micron contains sharp diffraction peaks revealing the large size of the crystals. Further the size and morphology of the amorphous and crystalline samples were evaluated using microscopy (SEM and TEM). The amorphous particles (Fig. 2a) have uniform size of 20-30 nm without sharp edge. Remarkably, spherical monodispersed nanosized crystals with sizes in the range of 30-45 $\mathrm{nm}$ were obtained for sample FAU-1-nano (Fig. 2b). The size of the crystals determined by SEM is consistent with the XRD results. As shown in Fig. 2c, the FAU-2-nano crystals have a diameter of 50-80 nm. While sample FAU-3-micron (Fig. 2d) contains crystals with a size of 500 $\mathrm{nm}$ and with pronounced octahedral-morphology. These results point out that via decreasing the concentration of sodium, bigger FAU crystals with more defined octahedral shape cane be synthesized. Further the crystalline nature, morphology and size of particles in the samples are revealed based on TEM. As shown in Fig. 3a, the amorphous particles with size of $25 \mathrm{~nm}$ do not show any crystalline fringes. While the lattice fringes corresponding to [111] plan in the samples FAU-1-nano (30-45 nm, FAU-2-nano $(50-80 \mathrm{~nm})$ and FAU-3-micron $(500 \mathrm{~nm})$ are clearly present (Fig. 3b, c, d).

The porosity of the amorphous and crystalline samples was evaluated by $\mathrm{N}_{2}$ physisorption measurements. As shown in the Fig. 4, the amorphous sample exhibits a combination of type I and IV isotherm containing a H1-type hysteresis. The high mesoporosity is attributed to the interparticle voids originated from packing of amorphous particles that delineate regular mesopores with diameters of $25 \mathrm{~nm}$. The amorphous precursor shows a feature of micropore filling at low $\mathrm{P} / \mathrm{P}_{0}\left(1 \times 10^{-7}\right.$ $1 \times 10^{-3}$ ), indicating the micropore formation during the aging process. FAU-1-nano, FAU-2-nano and FAU-3-micron exhibit high microporosity and in addition textural mesoporosity. The textural mesoporosity is associated with the close packing of monodispersed and well-shaped nanosized crystallites. The total pore volume, micropore volume, specific surface area, and the external surface area of the samples 
are summarized in Table 2. The results imply that the amorphous particles were transferred to FAU zeolite with well-developed porosity.

The transformation of the amorphous nanoparticles into FAU zeolite under treatment with different concentration of $\mathrm{NaOH}$ is followed by FTIR (Fig. 5). The FTIR spectra of faujasite zeolite samples contain two bans, the symmetric stretching band at $744 \mathrm{~cm}^{-1}$ and double six ring vibration at $566 \mathrm{~cm}^{-1}$ thus confirming the crystalline nature of the samples. In addition, the T-O-T asymmetric vibration for samples FAU-1-nano, FAU-2-nano and FAU-3-micron appear at $983 \mathrm{~cm}^{-1}, 995 \mathrm{~cm}^{-1}$ and $1003 \mathrm{~cm}^{-1}$, respectively implying that the $\mathrm{Si} / \mathrm{Al}$ ratio increases.

The loss of zeolite-type water from the amorphous precursor is about $15.7 \%$ as determined by TG (Fig. 6). While the weight losses of FAU-1-nano, FAU-2-nano and FAU-3-micron are 21.8\%, 22.8\% and 16\%, respectively. Compared with the FAU-3-micron, samples FAU-1-nano and FAU-2-nano contain more water due to the smaller crystal sizes and higher Al content as shown by ICP and IR characterizations. Further the chemical composition and the silica arrangement in the faujasite samples are determined by ${ }^{29} \mathrm{Si}$ NMR. As shown in the Fig. 7, the amorphous precursor after aging $24 \mathrm{~h}$ at room temperature present a broad peak around 90 ppm typically observed for amorphous silica materials. From the ${ }^{29} \mathrm{Si}$ NMR of sample FAU-1-nano the $\mathrm{Si} / \mathrm{Al}$ ratio of 1.1 is calculated, which is consistent with the ICP result. The spectrum of sample FAU-2-nano contains five bands at 84.8, 89.5, 94.2, 99.3 and 103 ppm (Fig. 7c). The Si/Al ratio calculated for sample FAU-2-nano is 1.5, while for FAU-3-micron is 1.7 (Fig. 7d); five bands at 83.7, 88.1, 93.4, 98.4 and $101.1 \mathrm{ppm}$ in the ${ }^{29} \mathrm{Si}$ NMR spectra are present for both samples with slight difference in their intensity. The NMR and ICP study indicate that a higher $\mathrm{Na}_{2} \mathrm{O} / \mathrm{Al}_{2} \mathrm{O}_{3}$ ratio in the amorphous mixture favors the formation of FAU-1-nano with a crystalline yield of $85 \%$ after a shorter crystallization time, while the lower $\mathrm{Na}_{2} \mathrm{O} / \mathrm{Al}_{2} \mathrm{O}_{3}$ ratio of the precursor mixture produces FAU-2-nano and FAU-3-micron crystals with a higher yield of $90 \%$ after prolonged crystallization time.

The crystallization process of FAU-2-nano and FAU-3-micron was studied by X-ray diffraction. As shown in Fig. S1, the weak diffraction peak located at $6.11^{\circ}$ belonging to FAU zeolite can be found in the pattern of sample FAU-2-nano after 22 h. While after $24 \mathrm{~h}$, about $60 \%$ of the amorphous precursor is transform to faujasite zeolite. After $36 \mathrm{~h}$, the amorphous phase completely transformed to zeolite crystals. The SEM images corresponding to these samples are shown in Fig. S2. The sample 
treated for $22 \mathrm{~h}$ contains few particles with a size of $40 \mathrm{~nm}$. After extension of the crystallization time to $24 \mathrm{~h}$, the particles became with sharper edges. While after $36 \mathrm{~h}$, the amorphous particles transform into crystals of size of 50-80 nm. The porosity of these samples was measured by $\mathrm{N}_{2}$ physisorption (Fig. S3 and Table S1). The microporous specific surface area of the amorphous precursor is $85 \mathrm{~m}^{2} / \mathrm{g}$, whereas, the microporous specific surface area for sample after $20 \mathrm{~h}$ treatment is $19 \mathrm{~m}^{2} / \mathrm{g}$. The microporous specific surface area of samples after crystallization for $22 \mathrm{~h}$ and $24 \mathrm{~h}$ are $31 \mathrm{~m}^{2} / \mathrm{g}$ and $542 \mathrm{~m}^{2} / \mathrm{g}$, respectively, and for the fully crystalline sample FAU-2-nano is $875 \mathrm{~m}^{2} / \mathrm{g}$. Similar trends were observed for the other samples. The XRD and SEM results for sample FAU-3-micron are shown in Fig. S4-S5. Similar results were observerd except the kinetics of crystal growth. After crystallization for $8 \mathrm{~d}$ only $10 \%$ crystalline product was obtained. The crystals exhibit octahedral morphology with a size in the range $100-250 \mathrm{~nm}$. About $65 \%$ of the amorphous precursor is transform to FAU crystals with a size of $400 \mathrm{~nm}$ after $9 \mathrm{~d}$. All amorphous precursor nanoparticles are transformed to FAU after $11 \mathrm{~d}$. The evolution of porous structure in the FAU-3-micron series is followed by $\mathrm{N}_{2}$ and the results are shown in Fig. S6 and Table S2. The results are in a good agreement with the XRD and SEM.

The sodium cations act as inorganic template and ensure the high degree of supersaturation of the suspensions. The high concentration of sodium in the suspensions lead to the formation of small nanosized crystals with uniform particle size distribution (samples FAU-1-nano and FAU-2-nano). While the lower sodium content leads to the formation of bigger crystals (sample FAU-3-micron). During the crystal growth of sample FAU-3-micron, the low concentration of sodium penetrates in the amorphous precursor particles slower. Then large amount nuclei are formed and grow via aggregation due to the limited amount of sodium in the system. The significant decrease in particle size from FAU-2-nano and FAU-3-micron to FAU-1-nano indicates that short crystallization time and high concentration $\mathrm{NaOH}$ facilitate the formation of mono-disperse nanosized crystals, while the lower $\mathrm{Na}^{+}$ amount and longer crystallization time are beneficial for the formation of larger particles.

The XRD patterns of the three fully crystalline samples of FAU-1-nano, FAU-2-nano and FAU-3-micron in H-form are shown in Fig. S7. The high crystallinity of these samples indicates their stability after ion-exchange. Besides, a gradually increased of medium strong acid cites is observed. The change peaks at 201 
${ }^{\circ} \mathrm{C}, 232{ }^{\circ} \mathrm{C}$ and $250{ }^{\circ} \mathrm{C}$ in the $\mathrm{NH}_{3}$-TPD profiles of FAU-1-nano, FAU-2-nano and FAU-3-micron, respectively are shown in Fig. S8. Dealkylation of 1,3,5-triisopropylbenzene (TiPBz, a kinetic diameter of $0.95 \mathrm{~nm}$ larger than kinetic diameter of FAU of $0.74 \mathrm{~nm}$ ) is selected as a model reaction to characterize the samples FAU-1-nano, FAU-2-nano and FAU-3-micron. As shown in Fig. 8, the conversions of TiPBz on FAU-1-nano, FAU-2-nano and FAU-3-micron are 10\%, $55 \%$ and $10 \%$, respectively. The conversion of FAU-2-nano is much higher than that of FAU-1-nano and FAU-3-micron at the initial stage of the reaction; the conversion is maintained $20 \%$ higher for sample FAU-2-nano even after 160 min of reaction time. The results of $\mathrm{TiPBz}$ dealkylation indicates that the enhanced catalytic performance of sample FAU-2-nano is originated from the decreased crystal size and increased $\mathrm{Si} / \mathrm{Al}$ ratio. The FAU-1-nano has the highest external surface areas (230 $\mathrm{m}^{2} / \mathrm{g}$ ) but weaker acidity. On the contrary, the FAU-3-micron sample has strong acidity but low external surface area $\left(72 \mathrm{~m}^{2} / \mathrm{g}\right)$.

\section{Conclusion}

In summary, controlling the amount of sodium cation is the key to control both the nucleation and growth processes of FAU zeolite using the two-step synthesis approach. The amorphous nuclei with high Si/Al ratio were obtained under the growth-limiting nutrient $\left(\mathrm{Na}^{+}\right)$at room temperature followed by crystallization at 50 ${ }^{\circ} \mathrm{C}$ for different times. The FAU-1-nano zeolite with nanosized crystals of 30-45 nm and $\mathrm{Si} / \mathrm{Al}$ ratio of 1.1 was obtained for a short crystallization time and high $\mathrm{Na}^{+}$ concentration. On the contrary, larger crystals (the FAU-2-nano zeolite) with a dimension of $50-80 \mathrm{~nm}$ and $\mathrm{Si} / \mathrm{Al}$ ratio of 1.5 are obtained at lower sodium concentration. The FAU-3-micron crystals with a diameter of $500 \mathrm{~nm}$ and $\mathrm{Si} / \mathrm{Al}$ ratio of 1.7 were obtained for longer crystallization time and even lower sodium concentration. The FAU-2-nano crystals showed good catalytic performance due to the uniform nanosized crystals and high $\mathrm{Si} / \mathrm{Al}$ ratio. The $\mathrm{Si} / \mathrm{Al}$ ratio and crystal size of the FAU zeolites obtained by two-steps crystallization approach increase with a slight decrease of the concentration of sodium in the precursor suspensions.

\section{Conflicts of interest}

There are no conflicts to declare. 


\section{Acknowledgements}

The authors gratefully acknowledged, the National Natural Science Foundation of China (Grant No. U1862118, No. 21975285, No.21991091, No. 21991090, No. 21908246), the Fundamental Research Funds for the Central Universities (NO. 21CX06024A), and Postgraduate Innovation Projects (China University of Petroleum (East China)) (Grant No. YCX2021053). 


\section{References}

[1] Liang J., Shan G., Sun Y., Renew. Sust. Energ. Rev., 2021, 139, 110707

[2] Rong H., Wang G., Yan J., Zou X., Zhu G., Sci. China Mater., 2021, 64(2):374-382

[3] Hong M., Yu L., Wang Y., Zhang, J., Chen Z., Dong L., Zan Q., Li R., Chem.

Eng. J., 2019, 359: 363-372

[4] Kim J. Y., Oh H., Moon H. R., Adv. Mater., 2018, 31(20), 1805293

[5] Bacakova L., Vandrovcova M., Kopova I. Jirka I., Biomater. Sci., 2018, 6(5), 974-989

[6] Shen Y., Xu M., Li J., Qin Z., Wang C., Mintova S., Liu X., Inorg. Chem. Front., 2021, 8, 2144

[7] Sachse A., García-Martínez J., Chem. Mater., 2017, 29(9), 3827-3853

[8] Cheng X., Mao J., Lv X., Hua T., Cheng X., Long Y., Tang Y., J. Mater. Chem. A., 2014, 2(5), 1247-1251

[9] Rios C., Williams C., Fullen M., App. Clay Sci., 2009, 42, 446-454

[10] Novembre D., Di Sabatino B., Gimeno D., Pace C., Clay Miner., 2011, 46(3), 339-354

[11] Otero Areán C., Rodríguez Delgado M., Turnes Palomino G., Tomás Rubio M., Tsyganenko N. M., Tsyganenko A. A., Garrone E., Micro. and Meso. Mater., 2005, 80(1-3), 247-252

[12] Zhang G., Wang B., Zhang W., Li M., Tian Z., Dalton Trans., 2016, 45(15), 6634-6640

[13] Cho Y., Lee J., Bokare A. D., Kwon S.-B., Park, D.-S., Jung W.-S., Choi J.-S., Yang Y.-M., Lee J.-Y., Choi W., J. Ind. Eng. Chem., 2015, 22, 350-356

[14] Xu W, Zhang T, Bai R, Bai R., Zhang P., Yu J., J. Mater. Chem. A, 2020, 8(19), 9677-9683

[15] Arbel Haddad M., Ofer-Rozovsky E., Bar-Nes G., Borojovich E. J. C., Nikolski, D., Mogiliansky D., Katz A., J. Nucl. Mater., 2017, 493, 168-179

[16] Yokoi T., Yoshioka M., Imai H., Tatsumi T., Angew. Chem. Int. Ed., 2009, 48(52), 9884-9887

[17] Majano G., Darwiche A., Mintova S., Valtchev V., Ind. Eng. Chem. Res., 2009, 48(15), 7084-7091 
[18] Majano G., Delmotte L., Valtchev V., Mintova S., Chem. Mater., 2009, 21(18),4184-4191

[19] Iyoki K., Kamimura Y., Itabashi K., Shimojima A., Okubo T., Chem. Lett., 2010, 39(7), 730-731

[20] Goel S., Wu Z., Zones S. I., Iglesia E., J. Am. Chem. Soc., 2012, 134(42), 17688-17695

[21] Gatta G. D., Lee Y., Mineral. Mag., 2014, 78(2): 267-291

[22] Frising T., Leflaive P., Micro. Meso. Mater., 2008, 114(1-3), 27-63

[23] Rios C., Williams C., Fullen M., App. Clay Sci., 2009, 42(3-4), 446-454

[24] Guo H., Zhao L., Martineau-Corcos C., Fayon F., Viger-Gravel J., Awala H., Boullay P., Grand J., Vicente A., Gilson J.-P., Mintova S., Adv. Mater. Inter., 2021, 8(4): 2000634

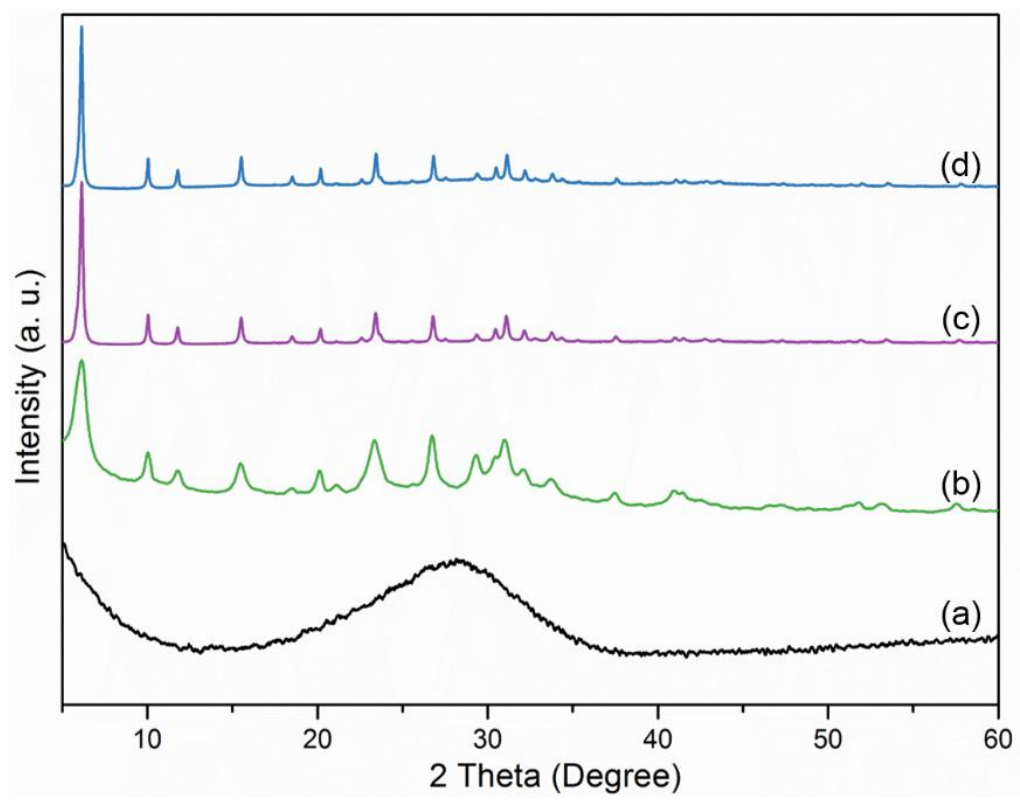

Fig. 1 XRD patterns of amorphous precursor (a), FAU-1-nano (b), FAU-2-nano (c) and FAU-3-micron (d). 


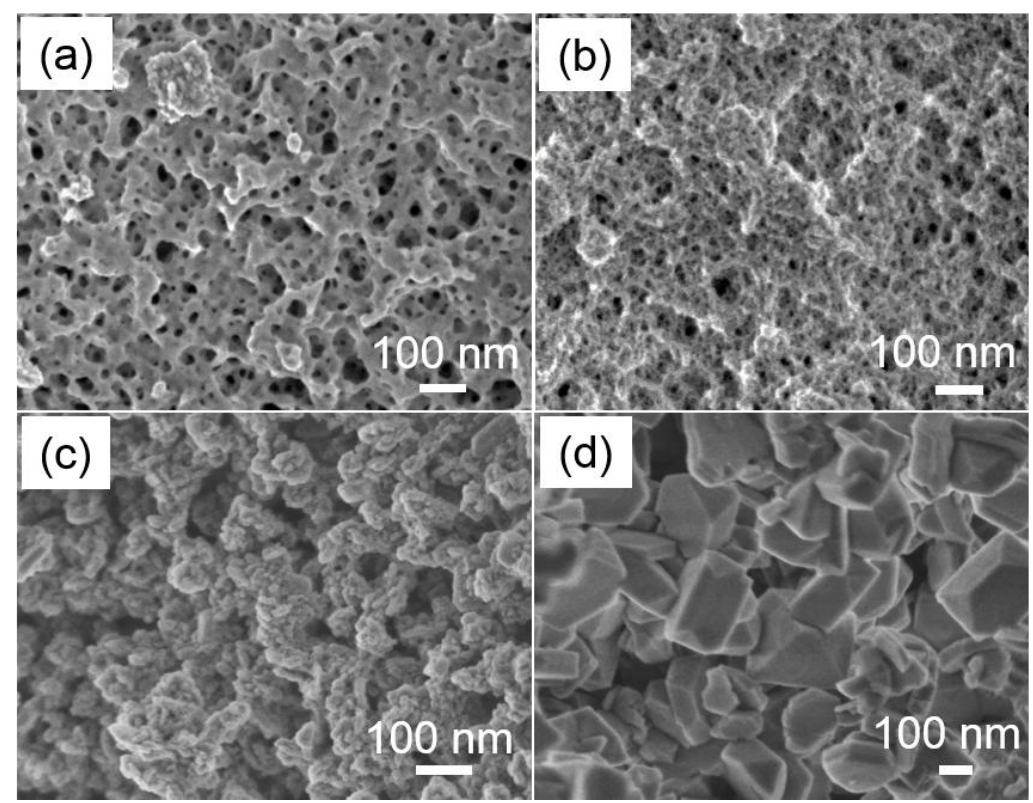

Fig. 2 SEM picture of amorphous precursor (a), FAU-1-nano (b), FAU-2-nano (c) and FAU-3-micron (d). 


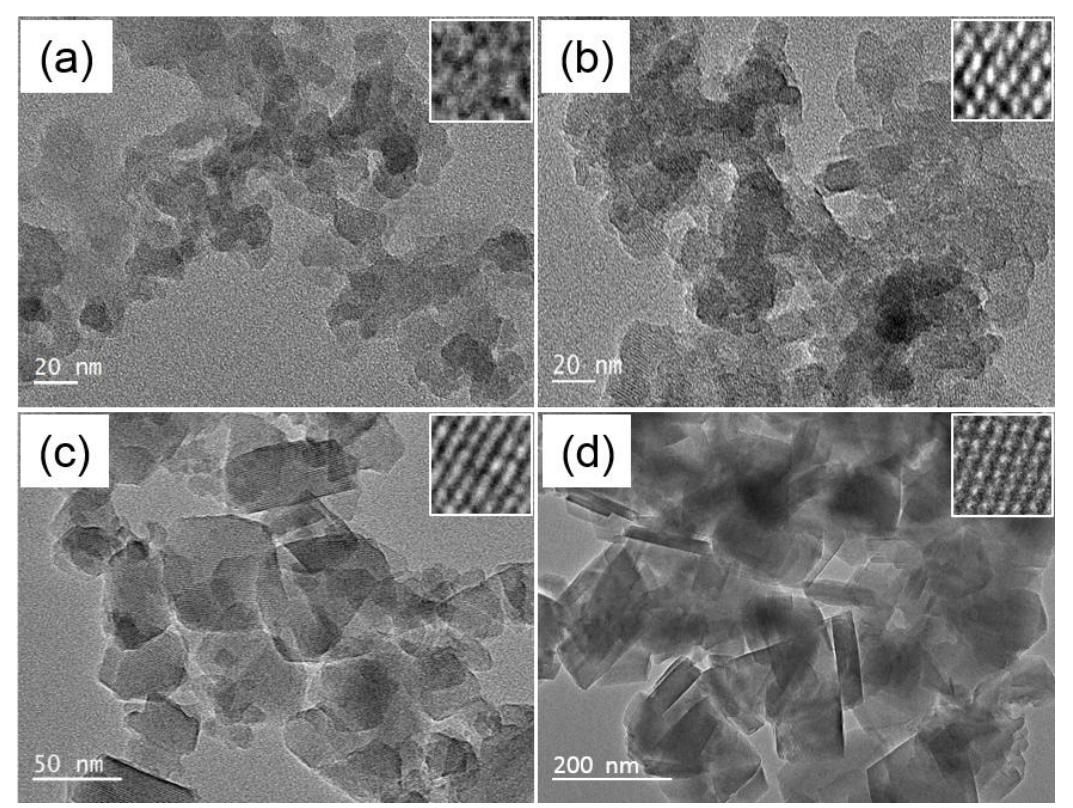

Fig. 3 TEM picture of amorphous precursor (a), FAU-1-nano (b), FAU-2-nano (c) and FAU-3-micron (d). 


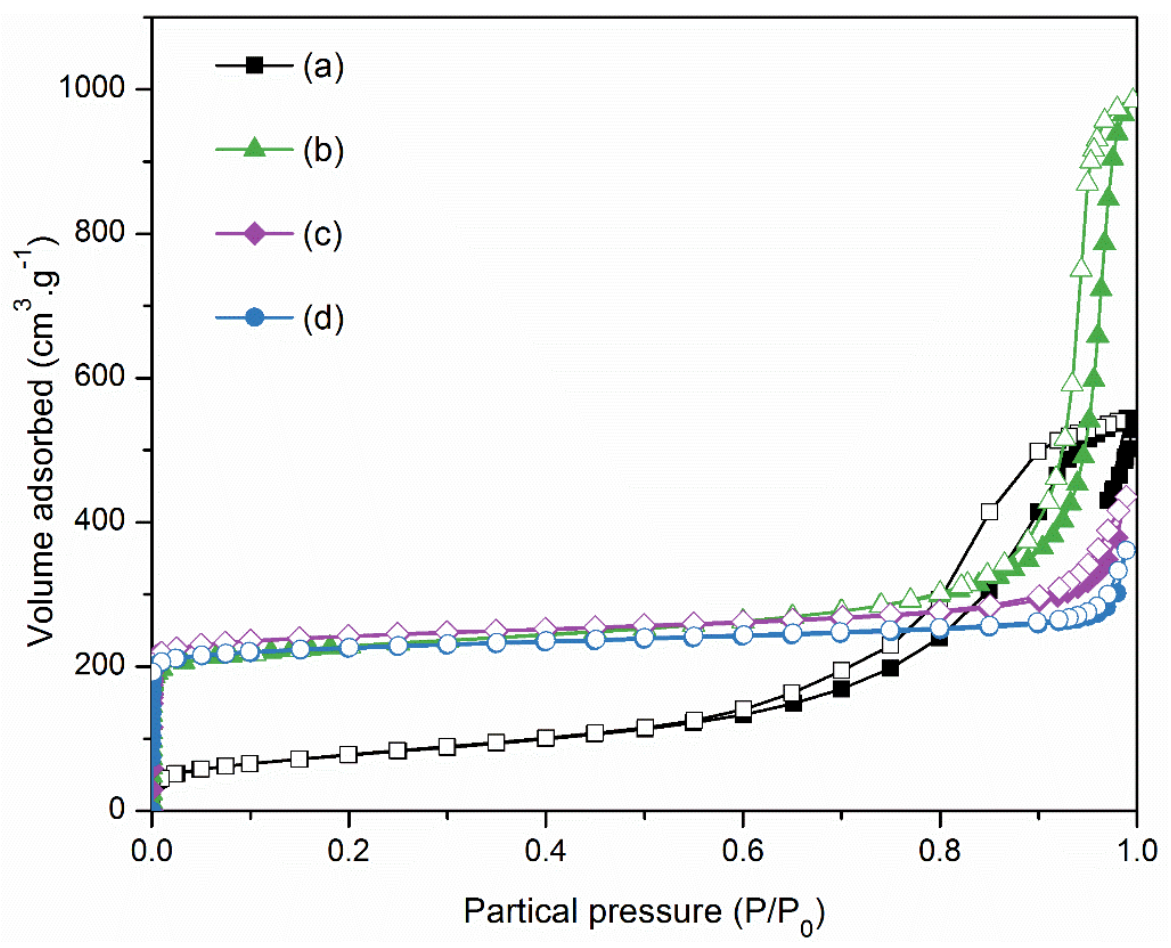

Fig. 4 Nitrogen sorption isotherms of amorphous precursor (a), FAU-1-nano (b), FAU-2-nano (c) and FAU-3-micron (d). 


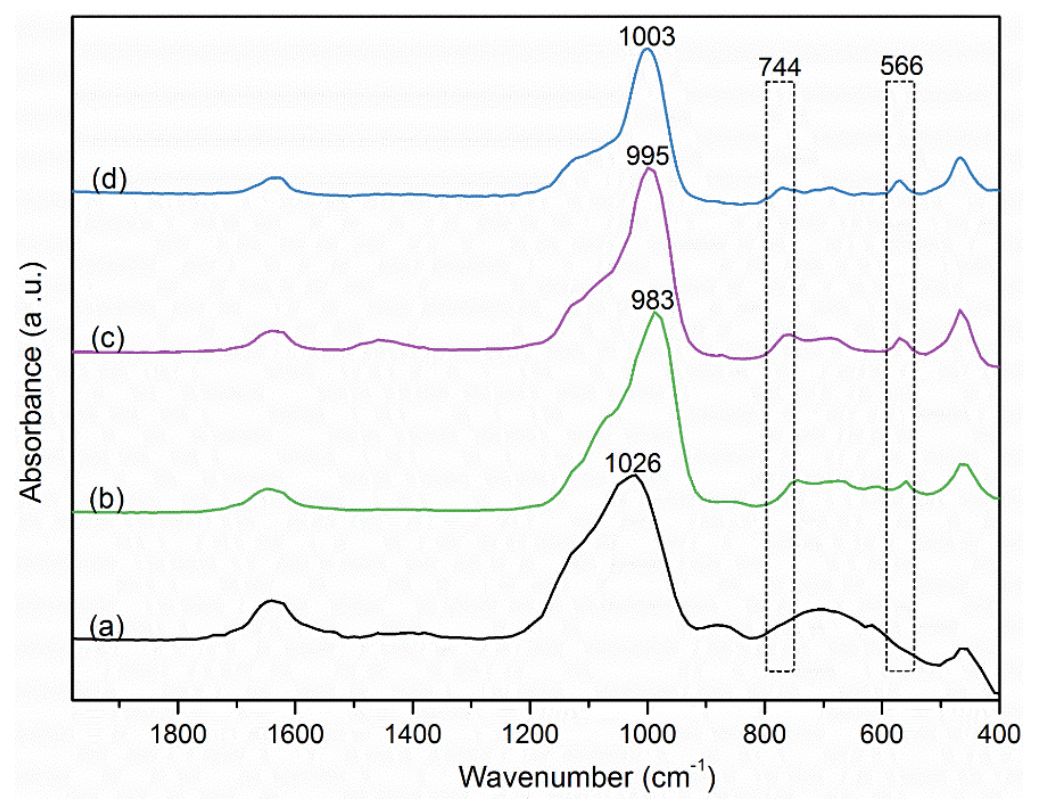

Fig. 5 FIIR patterns of amorphous precursor (a), FAU-1-nano (b), FAU-2-nano (c) and FAU-3-micro (d). 


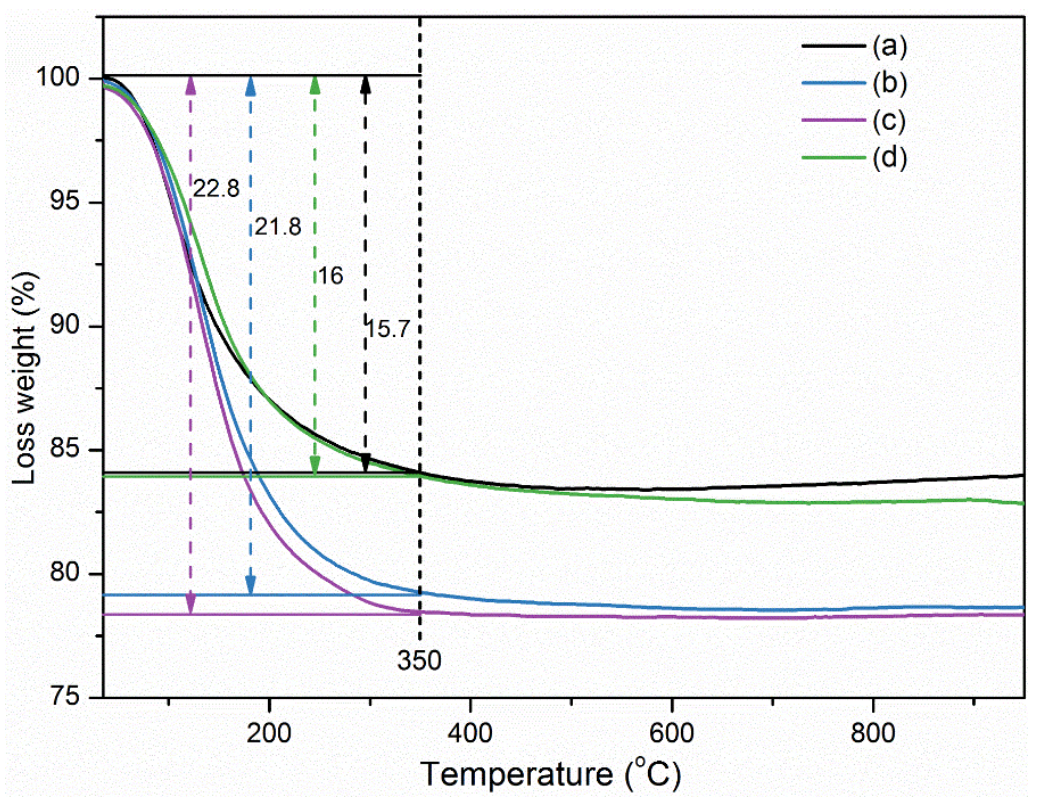

Fig. 6 TG curves of amorphous precursor (a), FAU-1-nano (b), FAU-2-nano (c) and FAU-3-micro (d). 

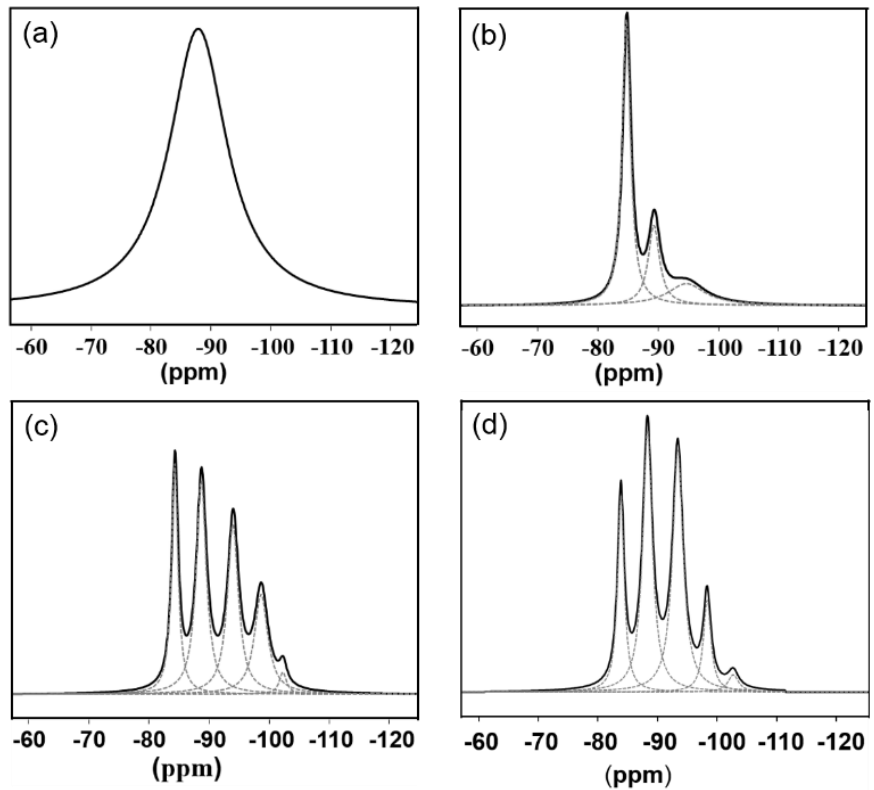

Fig. $7{ }^{29}$ Si NMR spectra of amorphous precursor (a), FAU-1-nano (b), FAU-2-nano (c) and FAU-3-micron (d). 


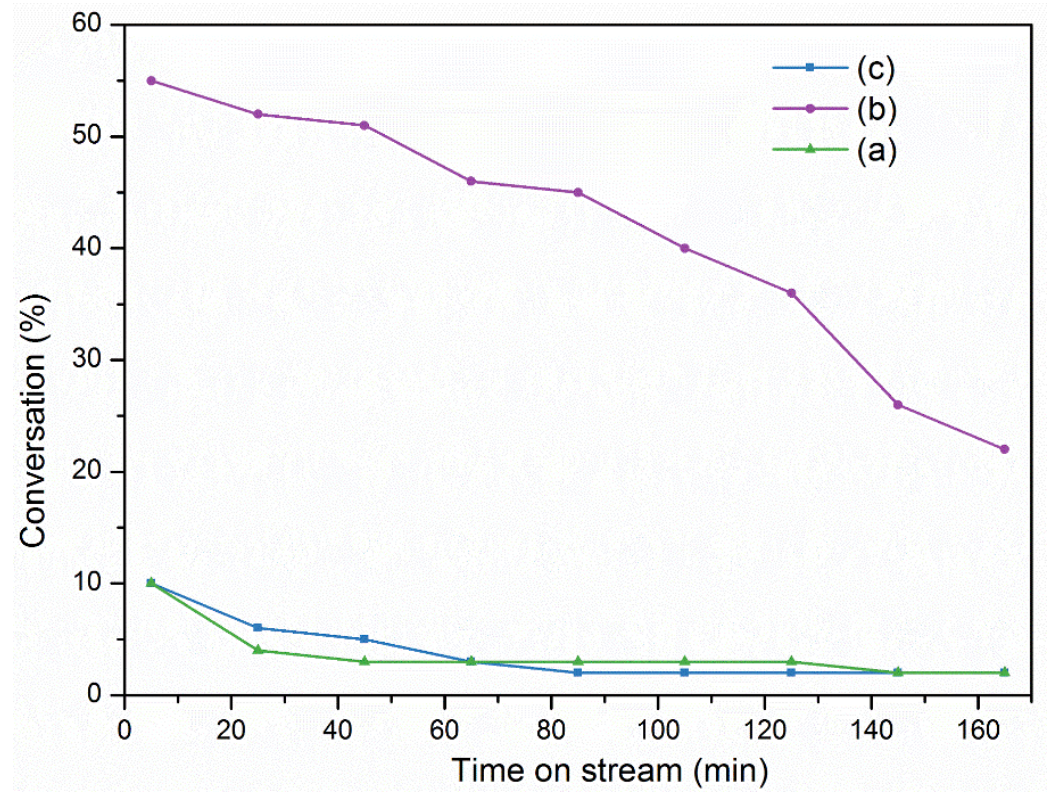

Fig. 8 Conversation for dealkylation on FAU-1-nano (a), FAU-2-nano (b) and FAU-3-micron (c) in H-form. 
Table 1. Transformation of amorphous precursors into crystalline FAU samples under treatment with $\mathrm{NaOH}$ with different concentrations and different crystallization conditions.

\begin{tabular}{cccc}
\hline Sample & $\begin{array}{c}\text { Concentration of NaOH } \\
(\mathrm{LiOH} / \mathrm{KOH})\end{array}$ & Solid/ Liquid ratio & $\begin{array}{c}\text { Synthesis } \\
\text { temperature } \\
\text { /time }\end{array}$ \\
\hline amorphous precursor & $0 \mathrm{wt} \%$ & -- & -- \\
FAU-1-nano & $7 \mathrm{wt} \% \mathrm{NaOH}$ & 0.05 & $50^{\circ} \mathrm{C} / 15 \mathrm{~h}$ \\
FAU-2-nano & $2 \mathrm{wt} \% \mathrm{NaOH}$ & 0.05 & $50{ }^{\circ} \mathrm{C} / 36 \mathrm{~h}$ \\
FAU-3-micron & $1 \mathrm{wt} \% \mathrm{NaOH}$ & 0.05 & $50^{\circ} \mathrm{C} / 11 \mathrm{~d}$ \\
\hline
\end{tabular}


Table 2 Physicochemical properties of amorphous, FAU-1-nano, FAU-2-nano, and FAU-3-micron.

\begin{tabular}{ccccccc}
\hline Sample & $\mathbf{S}_{\text {BET }}$ & $\mathbf{V}_{\text {mic }}$ & Smic & $\mathbf{V}_{\text {mes }}$ & $\mathbf{S}_{\text {ext }}$ & $\mathbf{V}_{\text {tot }}$ \\
& $\left(\mathbf{m}^{2} / \mathbf{g}\right)$ & $\left(\mathbf{c m}^{3} / \mathbf{g}\right)$ & $\left(\mathbf{m}^{2} / \mathbf{g}\right)$ & $\left(\mathbf{c m}^{3} / \mathbf{g}\right)$ & $\left(\mathbf{m}^{2} / \mathbf{g}\right)$ & $\left(\mathbf{c m}^{3} / \mathbf{g}\right)$ \\
\hline amorphous precursor & 175 & 0.01 & 85 & 0.32 & 90 & 0.33 \\
\hline FAU-1-nano & 850 & 0.31 & 620 & 1.05 & 230 & 1.36 \\
\hline FAU-2-nano & 985 & 0.34 & 859 & 0.17 & 126 & 0.67 \\
\hline FAU-3-micron & 897 & 0.32 & 825 & 0.08 & 72 & 0.40 \\
\hline
\end{tabular}

